\title{
COMPUTER CHESS REVIEWS
}

\author{
Tony Marsland
}

The primary aim of this column is to condense and review progress in computer chess. To this ,end readers are encouraged to submit summaries of their recent results to T. A. Marsland, Computing Science Department, University of Alberta, Edmonton T6G 2H1, Canada.

More Chess and Computers, D. Levy and M. Newborn, Computer Science Press, 1980 , 117 pages.

There is something here for everybody. For the player, a set of computer chess games with analyses; for the programmer, data on how fast chess programs evaluate positions; and for the researcher, a theoretical problem of some interest. There has been rapid progress in the computer chess field over the past five years, reflected in the fact that a new edition of this book is planned, containing not only a discussion of the microcomputer revolution and the decade of work which followed the "Levy Challenge", but also an assessment of the current computer chess champion, Belle.

More Chess and Computers opens by reviewing how, in 1968, established scientists were drawn into substantiating their faith in artificial intelligence with more than words. Although International Master David Levy had no difficulty in winning his challenge in 1978 by reasserting his dominance over Chess 4.7 , there were now several programs of high caliber. That significant progress had been made was apparent in 1977 when Belle demonstrated its capabilities at the IFIP Conference in Toronto by successfully defending with a King and Rook against some of North America's best chess players using King and Queen. The program's strategy was so different from conventional thinking that the masters were unable to win in the required fifty moves. This book surveys all the leading programs of the time and illustrates their play with several annotated games.

One should not be misled into thinking that the book is just a typical collection of chess tournament games, as its predecessors tended to be. It also gives a detailed discussion of positions which current programs may not be able to handle. These positions illustrate that humans are very good at breaking seemingly complex situations into a set of simpler components, and recognizing that if certain structures (conditions) remain intact, then the components remain independent of each other and hence that the complex problem is in fact simple. Most chess programs do not have this analytic capability (i.e., do not have intelligence) but can in fact solve such problems if the number of available moves is quite small, so that each continuation can be examined quite deeply. As a consequence, the authors hypothesize "it is quite possible that deeper searches of the game tree will result in performances that asymptotically approach some fixed level"; however, "there is nothing to prove...this... will be at World Championship class". 
The book addresses another problem in the field, namely, computer-aided chess. The authors report on an experiment, proposed by Daniel McCracken, to determine whether "a chess program working in cooperation with a human could produce a combined playing strength greater than either of them individually". Although the first public experiment of this type did not demonstrate such successful symbiosis, there is much hope here since, as McCracken pointed out, "in a number of areas man and machine function better as a pair", and certainly most computer scientists would like to think so. One general conclusion was that "for man and machine to work together, considerable planning is necessary to take best advantage of the machine".

Despite the fact that the book spends some time discussing scientific aspects of the computer chess field, one feels that the authors, like the organizers of micro-computer chess events, have a long way to go before they meet the goals of John McCarthy, who stresses "the importance of viewing the tournament as a scientific experiment as well as a competitive event". Apart from this weakness, however, the book makes interesting reading, covering not only a history of the field for the decade of the 1970 's but also a good tabulation of the major computer chess programs and their authors. Although major portions of the book are primarily of interest to the chess enthusiast, there is more than enough to satisfy the general reader. For aspiring researchers there is even an interesting section describing an unsolved problem which arises out of the principal algorithm in computer chess programs. 